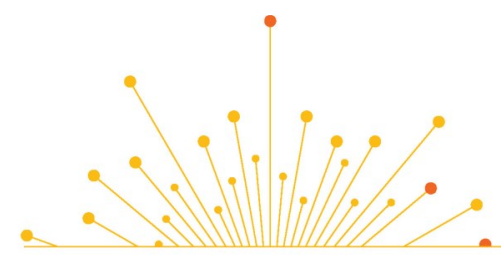

SCIENCE TABLE

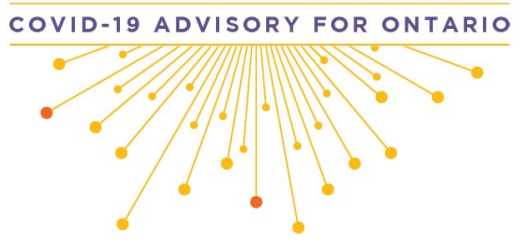

Version 1.0

Published: April 19, 2021

Citation: Morris AM, Bean S, Bell CM, et al. Strategies to manage tocilizumab supply during the COVID-19 pandemic. Science Briefs of the Ontario COVID-19 Science Advisory Table. 2021;2(22). https:// doi.org/10.47326/ocsat.2021.02.22.1.0

Author Affiliations: The affiliations of the members of the Ontario COVID-19 Science Advisory Table can be found at https:// covid19-sciencetable.ca/.

Declarations of Interest: The declarations of interest of the members of the Ontario COVID19 Science Advisory Table, its Working Groups, or its partners can be found at https://covid19sciencetable.ca/. The declarations of interest of external authors can be found under additional resources at https:// doi.org/10.47326/ocsat.2021.02.22.1.0.

About Us: The Ontario COVID-19 Science Advisory Table is a group of scientific experts and health system leaders who evaluate and report on emerging evidence relevant to the COVID-19 pandemic, to inform Ontario's response. Our mandate is to provide weekly summaries of relevant scientific evidence for the COVID-19 Health Coordination Table of the Province of Ontario, integrating information from existing scientific tables, Ontario's universities and agencies, and the best global evidence. The Science Table summarizes its findings for the Health Coordination Table and the public in Science Briefs.

The Drugs \& Biologics Clinical Practice Guidelines Working Group is a group of clinicians and scientists with recognized expertise in drugs, biologics, and clinical care. The Working Group evaluates existing scientific data, disease epidemiology, drug availability, and implementation issues in order to develop Clinical Practice Guidelines for the treatment of COVID-19 using drugs and biologics. The Working Group reports its findings to the public and the Science Table. Its findings are also summarized in Science Briefs.

Correspondence to: Secretariat of the Ontario COVID-19 Science Advisory Table
SCIENCE BRIEFS

\section{Strategies to Manage Tocilizumab Supply During the COVID-19 Pandemic}

Andrew M. Morris, Sally Bean, Chaim M. Bell, Martin Betts, Jennifer Gibson, Christopher Graham, Rebecca Greenberg, Peter Jüni, Julie Ann Justo, Bradley Langford, Elizabeth Leung, Erin K. McCreary, Laveena Munshi, Srinivasan Murthy, Sumit Raybardhan, Nathan M. Stall, Robert Steiner, Menaka Pai on behalf of the Drugs \& Biologics Clinical Practice Guidelines Working Group and the Ontario COVID -19 Science Advisory Table

\section{Key Message}

Tocilizumab is an anti-inflammatory medication that acts by inhibiting interleukin- 6 (IL-6) and is shown to improve outcomes including mortality in patients hospitalized with COVID-19 requiring supplemental oxygen.

Ontario supply of tocilizumab is limited, and tocilizumab demand in Ontario might exceed supply in the near future.

A strategy that includes using a fixed, single intravenous dose of $400 \mathrm{mg}$ for eligible patients will help extend available supply and is likely effective in treatment of COVID19. Sarilumab, another IL- 6 inhibitor, can be considered as a substitute. Additional options to consider to optimize tocilizumab use include the use of a provincial dashboard to help monitor and allocate use and estimating supply-to-demand adequacy. Likewise, a centralized allocation lottery system could be employed as soon as predicted demand exceeds supply to help ensure fair allocation. However, other issues may need to be taken into account for allocation decisions, as appropriate.

\section{Lay Summary}

Patients with the most severe cases of COVID-19 develop dangerous inflammation that can compromise their lungs and other vital organs. As a COVID-19 treatment, tocilizumab is used in hospitalized patients whom clinicians determine to be "moderately" to "critically" ill with COVID-19 and who have not yet responded to other standard medical care. Patients receiving tocilizumab should already be receiving corticosteroids (a class of drugs that also reduce inflammation) and oxygen.

Prior to the COVID-19 pandemic, tocilizumab was used for a few uncommon medical conditions. Research studies have demonstrated that tocilizumab has the ability to save lives in people hospitalized with COVID-19 who are moderately or severely ill. This has meant a large increase in global demand for tocilizumab that is greater than existing supply. Ontario is facing a similar situation.

By reducing the dose of tocilizumab by up to half from what was studied and to only use a single dose (in studies, about one third of patients received a second dose), we believe that we can continue to offer the same chance of each eligible COVID-19 patient benefiting from treatment while substantially increasing the number of patients who receive it. Sarilumab is a drug that acts similar to tocilizumab that is less well studied in COVID-19 but might be a reasonable substitute during critical shortages.

An important option for consideration - if shortages are likely - is the establishment 
of a system that ensures tocilizumab is distributed in a way that gives every COVID19 patient who needs tocilizumab an equal chance of receiving it, regardless of where they live, the hospital delivering treatment, or the province's remaining supply. If necessary, this might mean that a lottery would be used to make sure the distribution is as fair as possible.

\section{Summary}

\section{Background}

There is high quality evidence that tocilizumab, an interleukin- 6 inhibitor, saves lives in patients recently hospitalized with COVID-19 who require supplemental oxygen or ventilatory support. In Ontario, the Critical Care COVID-19 Command Centre has established the Provincial ICU Drug Task Team to monitor and support drug supply for critically ill COVID-19 patients, including tocilizumab.

There is currently a worldwide shortage of tocilizumab due to increased demand for both on-label indications (e.g., refractory rheumatologic disorders) and off-label indications (e.g., COVID-19), as well as limited production capability. This has put a strain on the allocation system and provincial supply, and existing supply might not be sufficient. Hospitals have independently adopted various approaches to rationing, not all of which are evidence-based. Variability in centre-to-centre use of tocilizumab suggests there may also be inequities in access to this medication based on geography, depending on where COVID-19 patients present for initial care.

During Ontario's third wave of COVID-19 there is an increasing number of patients hospitalized with COVID-19 and eligible for treatment with tocilizumab, which poses a challenge for allocation and distribution of a medication that is a scarce resource. To date, there has been no clear risk-based approach to allocating tocilizumab both among and within Ontario hospitals during the pandemic. A Science Brief on sarilumab, another IL-6 inhibitor that could act as a substitute for tocilizumab, is currently in preparation, but early considerations for sarilumab are provided below.

\section{Questions}

Can we further optimize dosing of tocilizumab for COVID-19 patients to conserve supply?

Can we substitute tocilizumab with sarilumab for the treatment of COVID-19?

Are most COVID-19 patients who would benefit from tocilizumab receiving it?

How could we optimize the distribution and supply of tocilizumab to ensure the most benefit?

\section{Findings}

Dosing of tocilizumab for COVID-19 ( $8 \mathrm{mg} / \mathrm{kg}$, with consideration of a second dose after 24 hours if patients do not show clinical improvement), as recommended in our original Science Brief, was based on published randomized controlled trials. However, pharmacokinetic data and expert opinion suggest that alternate dosing strategies could conserve the limited supply of tocilizumab while conferring therapeutic benefit for treatment of COVID-19.

Tocilizumab is currently used for its inhibitory effects on IL-6 receptors at doses ranging from $4 \mathrm{mg} / \mathrm{kg}$ to $8 \mathrm{mg} / \mathrm{kg}$. Most pharmacokinetic studies of tocilizumab are not necessarily applicable to the treatment of COVID-19 patients. IL-6 levels in COVID-19 are significantly lower than in cytokine release syndrome in other clinical situations. 
The half-life of tocilizumab after one dose is prolonged. Although roughly $30 \%$ of patients received a second dose of tocilizumab in RECOVERY and REMAP-CAP, the two largest RCTs that showed benefit from tocilizumab, neither trial provides information sufficient to understand the role of a second dose in patients who did not respond to a first dose.

Tocilizumab is supplied in $80 \mathrm{mg}, 200 \mathrm{mg}$ and $400 \mathrm{mg}$ vials. Hospitals which, for example, only have access to $400 \mathrm{mg}$ vials may waste tocilizumab by administering a $600 \mathrm{mg}$ dose to a using weight-based dosing at $8 \mathrm{mg} / \mathrm{kg}$. By keeping the dosing consistently at $400 \mathrm{mg}$, there would be no anticipated wastage.

Sarilumab is a fully human monoclonal antibody that, like tocilizumab, also targets both soluble and membrane-bound IL-6 receptors. In the largest trial to show a mortality benefit for IL- 6 therapies in COVID-19, sarilumab was given as a single 400 $\mathrm{mg}$ intravenous infusion and has been recently reported as equivalent to tocilizumab. Sarilumab is only available as a subcutaneous formulation on the Canadian market and is not approved for intravenous administration. However, the subcutaneous formulation has been compounded for intravenous administration in study protocols and could be used in many Ontario hospitals.

Challenges to tocilizumab access and utilization are related to distribution processes and logistics, whereas others are related to knowledge translation. Several hospitals in Ontario have recently run out of tocilizumab supply and have been unable to obtain additional amounts through informal regional sharing.

\section{Practical Considerations}

Roche Canada is the sole supplier of tocilizumab in Canada. Tocilizumab is currently allocated to provinces and territories on a monthly basis. As hospitals face rising tocilizumab needs due to the surge of patients with COVID-19 in Ontario's current third wave, there is a potential that tocilizumab demand might exceed supply in the coming weeks based on projected hospitalizations and ICU admissions.

An ethical, evidence-based framework is required to inform allocation and use of limited tocilizumab supply. The Ontario COVID-19 Bioethics Table adapted a published Ontario drug supply shortage framework for use during the COVID-19 pandemic, that outlines a set of guiding ethical principles and three stages for managing drug supply during the COVID-19 pandemic:

Stage 1: Preserve the standard of care for as many COVID-19 patients as possible by a) conserving supply, b) sharing supply, and c) procuring or accessing new supply.

Stage 2: Optimize therapeutic benefit based on existing evidence within available supply (Primary Allocation Principles).

Stage 3: Use a fair procedure to choose between patients (Secondary Allocation Principle) if Stage 1 and 2 efforts are insufficient to meet demand.

\section{Recommendations}

If Ontario enters a shortage of Tocilizumab, it will need to consider approaches that optimize the effectiveness and equity of the existing supply. Working from the Ontario COVID-19 Bioethics Table framework noted above, this Science Brief provides recommendations and options at each stage.

\section{Recommendations for All Stages of Managing Tocilizumab Supply and Distribution}

- Ontario should continue to preserve tocilizumab for existing indications (e.g., refractory rheumatological disorders and chimeric antigen receptor (CAR) T-cell 
therapy for some hematologic cancers).

- Use of tocilizumab for COVID-19 patients should follow the Ontario COVID-19 Science Advisory Table's Clinical Practice Guidelines, which are supported by randomized controlled trials.

- Tocilizumab should be allocated province-wide to optimize therapeutic benefit and ensure equitable access for all eligible COVID-19 patients in Ontario.

\section{Options for All Stages of Managing Tocilizumab Supply and Distribution}

- The assessment of the stage of drug shortage should be based on predicted demand and communicated clearly to all clinical stakeholders.

- Ontario could create a near real-time provincial dashboard accessible to all clinical stakeholders that includes currently available tocilizumab supply, and allocated and administered doses (by region and site), which could also include any anticipated shipments of tocilizumab.

- Tocilizumab procurement could be provincial to support assessment of supply and management of supply.

- There could be a provincially coordinated mechanism, building on the Incident Management Systems (IMS), to allow transfer of medication (or, rarely, patients) to ensure that any patient admitted to a hospital lacking tocilizumab supply can receive timely, evidence-based therapy.

\section{Recommendations for Stage 2 of Tocilizumab Supply and Distribution}

- The dose of tocilizumab should be reduced in line with the evidence in this brief.

- Triaging of patients based on factors demonstrating which patients are most likely to benefit from treatment with tocilizumab has not been identified in the literature and is not recommended.

\section{Options for Stage 2 of Tocilizumab Supply and Distribution}

- Distribution of tocilizumab could be weekly and site-specific, with allocation based on the prior week's consumption.

\section{Recommendations for Stage 3 of Managing Drug Shortages}

- A logic-based mechanism to fairly ration tocilizumab should be developed.

- Patients eligible for tocilizumab allocation are either moderately ill hospitalized patients (i.e., requiring supplemental oxygen via nasal prongs) or critically ill hospitalized patients (i.e., requiring oxygen via venturi mask, high-flow nasal cannula, non-invasive mechanical ventilation, invasive mechanical ventilation, or extra-corporeal membrane oxygenation (ECMO)) with suspected or confirmed COVID-19 who are on recommended doses of dexamethasone (or another doseequivalent corticosteroid) and are within 14 days of hospital admission or within 14 days of a new COVID-19 diagnosis if acquired in a healthcare setting. Moderately ill patients should have additional evidence of systemic inflammation, defined as a C-reactive protein (CRP) of $75 \mathrm{mg} / \mathrm{L}$ or higher, and have evidence of disease progression (i.e., increasing oxygen or ventilatory requirements) while on dexamethasone or another dose-equivalent corticosteroid for treatment of COVID-19. Moderately ill COVID-19 patients at hospitals not offering CRP measurement should be considered for tocilizumab allocation.

- Tocilizumab should be given to COVID-19 patients with a reasonable chance of 
benefiting from the drug.

- A strictly first-come, first-served approach is not recommended.

- COVID-19 patients who meet the eligibility criteria but do not want active medical treatment or specific treatment with tocilizumab should neither be offered it nor entered into an allocation system.

Options for Stage 3 of Tocilizumab Supply and Distribution

- To mitigate potential bias influencing the assessment of COVID-19 patients eligible for tocilizumab, a second-opinion or consensus model among those responsible for assessing eligibility should be considered.

- An allocation lottery could be the fair default procedure for choosing among eligible COVID-19 patients where allocation based on maximizing benefits cannot be determined.

- If implemented, the allocation lottery should be initiated and managed centrally to avoid inequities in access due to geography.

- A committee should oversee the allocation lottery procedure, with representation of medical specialties prescribing tocilizumab, regional considerations, and patient and caregiver representation from communities most affected by COVID-19.

- An ideal allocation lottery would ensure meaningful and individualized access to tocilizumab for all COVID-19 patients, would avoid discrimination, and mitigate disparities in outcomes due to social inequalities. At this juncture, such a model is likely not feasible in Ontario. A simple unweighted lottery is therefore recommended.

- The first step to allocation lottery would be to predict the anticipated number of hospitalized COVID-19 cases and the available doses of tocilizumab over a defined time period, and to decide what percentage of eligible patients should receive tocilizumab.

- After patients are registered, the hospital official would receive treatment allocation following randomization, and would report the results of randomization to the prescriber and to the hospital pharmacy supplying tocilizumab. If the hospital does not have tocilizumab, they would notify the incident management system (IMS) to arrange delivery of a dose of tocilizumab.

- Implementing such a strategy would require careful communication. The communication strategy should include separate messaging to hospital administrators and healthcare leaders, healthcare providers, patients and their families, and the public (including scripts to support health care providers as they communicate with patients and their families).

- Patient-centred outcomes would be evaluated to assess the effectiveness of tocilizumab allocation and treatment during the shortage and under the new guidelines.

- Ideally, the allocation system could also be used to test therapeutic hypotheses (i.e., a pragmatic clinical trial comparing $400 \mathrm{mg}$ vs. $800 \mathrm{mg}$ tocilizumab dosing).

\section{Background}

There is high quality evidence that tocilizumab, an interleukin-6 inhibitor, saves lives in patients recently hospitalized with COVID-19 who require supplemental oxygen or 
ventilatory support. ${ }^{1}$

Tocilizumab is also used in refractory rheumatologic disorders, as well as in patients undergoing chimeric antigen receptor (CAR) T-cell therapy for some hematologic cancers. Stem Cell Transplant Centres in Ontario are required to stock four doses of tocilizumab prior to administering CAR-T cell therapy, in the event of a cytokine storm.

There is a federal, provincial, and territorial framework to manage drug shortages in Canada, enhanced during the COVID-19 pandemic, which also has developed a national framework for drug allocation. In Ontario, the Critical Care COVID-19 Command Centre has established the Provincial ICU Drug Task Team to monitor and support drug supply for critically ill COVID-19 patients. Throughout the pandemic, the ICU Drug Task Team has allocated tocilizumab to hospitals based on their recent usage; if hospitals need further stock, they can contact administrators for further allocation.

There is currently a worldwide shortage of tocilizumab due to increased demand for both on-label indications (e.g., cytokine storm and rheumatologic disorders) and offlabel indications (e.g., COVID-19), as well as limited production capability. This has put a strain on the allocation system and provincial supply of tocilizumab, and has led to hospitals being uncertain if their requests for additional stock will be met. Hospitals have independently adopted various approaches to rationing, not all of which are evidence-based. Variability in centre-to-centre use of tocilizumab suggests there may also be inequities in access due to geography, depending on where COVID -19 patients present for initial care.

During Ontario's third wave of COVID-19, the increasing number of patients hospitalized with COVID-19 and eligible for treatment with tocilizumab poses a challenge for the allocation and distribution of a medication that is a scarce resource. To date, there has been no clear risk-based approach to allocating tocilizumab both among and within Ontario hospitals during the pandemic.

A Science Brief on sarilumab, another IL-6 inhibitor that could act as a substitute for tocilizumab, is currently in preparation.

\section{Questions}

Can we further optimize dosing of tocilizumab for COVID-19 patients to conserve supply?

Can we substitute tocilizumab with sarilumab for the treatment of COVID-19?

Are most COVID-19 patients who would benefit from tocilizumab receiving it?

How could we optimize the distribution and supply of tocilizumab to ensure the most benefit?

\section{Findings}

\section{Can we further optimize dosing of tocilizumab for COVID-19 patients to conserve} supply?

Dosing of tocilizumab $(8 \mathrm{mg} / \mathrm{kg}$, with consideration of a second dose after 24 hours if patients do not show clinical improvement) as recommended in our original Science Brief, ${ }^{1}$ was based on published randomized controlled trials. However, pharmacokinetic data and expert opinion suggest that alternate dosing strategies could conserve the limited supply while conferring therapeutic benefit for treatment of COVID-19.

Key pharmacokinetic/pharmacodynamic findings include:

Tocilizumab is currently used for its inhibitory effects on IL-6 receptors in other 
indications, at a variety of doses including for rheumatoid arthritis $(4 \mathrm{mg} / \mathrm{kg}$ to $8 \mathrm{mg} /$ $\mathrm{kg}$ ), and cytokine release syndrome associated with chimeric antigen receptor (CAR) T-cell therapy $(8 \mathrm{mg} / \mathrm{kg})$.

Most pharmacokinetic studies of tocilizumab examined prolonged tocilizumab exposure in relatively stable patients with rheumatoid arthritis (e.g., 24 weeks).

Steady state maximum serum concentrations $\left(C_{\max }\right)$ and area under the curve (AUC) are achieved after a single intravenous dose with both a $4 \mathrm{mg} / \mathrm{kg}\left(C_{\max }=88 \mu \mathrm{g} / \mathrm{mL}\right.$, AUC $=13,000 \mu \mathrm{g} \cdot \mathrm{h} / \mathrm{mL})$ and $8 \mathrm{mg} / \mathrm{kg}\left(C_{\max }=183 \mu \mathrm{g} / \mathrm{mL}, A U C=35,000 \mu \mathrm{g} \cdot \mathrm{h} / \mathrm{mL}\right)$ dosing regimen. ${ }^{4}$

The steady-state half-life is expected to be longer than 7 days for most patients who received a $4 \mathrm{mg} / \mathrm{kg}$ dose; and up to 13 days with those receiving an $8 \mathrm{mg} / \mathrm{kg}$ dose, ${ }^{5}$ with limited data that drug clearance is impacted by weight. ${ }^{4}$

Studies demonstrate that lower concentrations of tocilizumab primarily block soluble (not membrane-bound) IL-6 receptor binding, thereby inhibiting the IL- 6 proinflammatory response while preserving its anti-inflammatory response. ${ }^{6,7}$

Pharmacokinetic simulations in rheumatoid arthritis patients, suggest that fixed dose regimens provide more consistent drug exposure across patient weight categories than weight-based dosing. These data indicate that a fixed dose may be a viable approach across a variety of weight ranges. ${ }^{8}$

The ideal pharmacokinetic/pharmacodynamic relationship between tocilizumab exposure and clinical outcomes in COVID-19 and other indications is unknown.

IL-6 levels in COVID-19 are significantly lower than in cytokine release syndrome associated with CAR T-cell therapy. ${ }^{7,9}$

Limited observational data have studied non-weight-based, lower doses of tocilizumab in COVID-19. For example, a study using doses of 40-200 mg showed a potential therapeutic effect but could not identify a dose-response relationship. ${ }^{10}$ Several trials of lower dose tocilizumab in COVID-19 are currently ongoing. ${ }^{11,12}$

Considerations regarding second doses are as follows:

The half-life of tocilizumab after one dose is prolonged. ${ }^{4,8}$ Although roughly $30 \%$ of patients received a second dose of tocilizumab in RECOVERY and REMAP-CAP, the two largest RCTs that showed benefit from tocilizumab, neither trial provides sufficient information to understand the role of a second dose in COVID-19 patients who did not respond to a first dose..$^{2,3}$

Operational considerations include:

The intravenous preparation of tocilizumab is supplied in $80 \mathrm{mg}, 200 \mathrm{mg}$ and $400 \mathrm{mg}$ single-use vials. Hospitals which only have access to $400 \mathrm{mg}$ vials, for example, would risk wasting tocilizumab by administering a $600 \mathrm{mg}$ dose using weight-based dosing at $8 \mathrm{mg} / \mathrm{kg}$. By keeping the dosing consistently at $400 \mathrm{mg}$, there would be no anticipated wastage. Single-use vials do not contain preservatives therefore, if not compounded under sterile preparation according to National Association of Pharmacy Regulatory Authorities (NAPRA) standards, vials must be discarded after single-use to prevent microbial contamination of the intravenous solution.

While there are observational data to support a lower fixed dose of tocilizumab (400 $\mathrm{mg}$ ) in the treatment of COVID-19, randomized controlled trials have not yet evaluated this approach. However, based on emerging evidence on lower IL-6 levels in COVID-19 patients, pharmacokinetic and pharmacodynamic considerations of IL-6 inhibition, the recommendation to use fixed dose tocilizumab has been made in the 
context of extremely limited drug supply and in an effort to maximize the number of individuals that could potentially benefit from the drug. In the absence of data showing the benefit of a second dose, we recommend that no second tocilizumab doses be offered to COVID-19 patients in a shortage situation.

\section{Can we substitute tocilizumab with sarilumab for the treatment of COVID-19?}

Sarilumab is a fully human monoclonal antibody that also targets both soluble and membrane-bound IL- 6 receptors, and has been studied in COVID-19 patients. While a class effect is plausible, the data are more robust for tocilizumab given the larger number of patients studied and the higher certainty of benefit compared to sarilumab. ${ }^{13}$ In the largest trial to show a mortality benefit for IL- 6 therapies in COVID19 patients, REMAP-CAP, sarilumab was given as a single $400 \mathrm{mg}$ intravenous infusion (12\% of the patients who received IL- 6 blockade therapy received sarilumab). ${ }^{3}$ Recently, the REMAP-CAP investigators reported that sarilumab and tocilizumab are equally effective for the treatment of patients with severe COVID-19, although data and a manuscript are unavailable at the time of this Science Brief's release. ${ }^{14}$ In critical shortages of tocilizumab, sarilumab may be considered for COVID-19 patients who meet criteria for IL-6 blockade and have not yet received any IL- 6 therapy.

Sarilumab is only available as a subcutaneous formulation on the Canadian market and is not approved for intravenous administration. ${ }^{15}$ However, the subcutaneous formulation has been compounded for intravenous administration in study protocols. Use of appropriate sterile technique (due to lack of preservatives in preparations), small volume manipulation, and short expiry of final diluted products are important considerations in compounded preparations of sarilumab for intravenous use.

\section{Are most COVID-19 patients who would benefit from tocilizumab receiving it?}

Roche Canada is the sole supplier of tocilizumab in Canada. The Canadian Pharmaceutical Distribution Network (CPDN) is the hospital distributor for Roche, and currently allocates the supply of off-label tocilizumab to provinces and territories on a monthly basis. The ICU Drug Task Team, in collaboration with Ontario's Ministry of Health and the University Health Network, subsequently distributes tocilizumab to Ontario hospitals based on criteria that include a hospital's COVID-19 ICU admissions and recent tocilizumab consumption. There is no real-time dashboard of tocilizumab supply available to Ontario hospitals. As hospitals face rising tocilizumab needs due to the surge of COVID-19 patients in Ontario's current third wave, there is a potential that tocilizumab demand might exceed supply in the coming weeks based on projected hospitalizations and ICU admissions. ${ }^{16}$

We surveyed pharmacy directors, clinical pharmacists, critical care physicians and general internal medicine physicians in 20 academic and community hospitals throughout Ontario during the week of March 9 to March 15, 2021, to better understand their recent experience with utilization and access to tocilizumab. The centres were purposefully chosen to represent a range of small and large centres across the province.

Two hospitals reported that their hospital's internal policy was currently more restrictive (e.g., limiting only to critically ill COVID-19 patients or limiting second dosing) than the Ontario COVID-19 Science Advisory Table's recommendation for tocilizumab use. ${ }^{1}$ The majority of surveyed centres reported infrequent administration of second doses of tocilizumab, including five centres $(25 \%)$ reporting local guidelines which discouraged second doses, mainly due to concerns around supply chain stability. 
Almost $30 \%$ of centres reported needing to access additional tocilizumab supply (above their allotment), however, not all centres were able to receive additional drug.

Some sites reported obtaining tocilizumab for COVID-19 treatment outside of provincial (i.e., CPDN) supply. Some sites reported not requesting drug from CPDN due to previously encountered unspecified difficulties in obtaining additional supply.

Some sites reported delays in tocilizumab administration due to delayed access to supply.

Clinicians expressed concern that some centres (particularly, smaller or rural centres) may seek to transfer COVID-19 patients for the express purpose of administration of tocilizumab for eligible patients, due to no local allocation or limited local experience or comfort around prescribing and administering tocilizumab.

\begin{tabular}{|c|c|}
\hline Principle & Description \\
\hline Beneficence & $\begin{array}{l}\text { Maintain highest quality of safe and effective care based on } \\
\text { available evidence and within available resources (i.e., drug supply) }\end{array}$ \\
\hline Equity & Ensure fair and non-discriminatory access to resources \\
\hline Reciprocity & $\begin{array}{l}\text { Respond to each other in similar ways that recognize mutual } \\
\text { interdependence }\end{array}$ \\
\hline Solidarity & $\begin{array}{l}\text { Build, preserve and strengthen inter-professional/-institutional/- } \\
\text { sectoral/-provincial collaborations and partnerships }\end{array}$ \\
\hline Stewardship & $\begin{array}{l}\text { Use available resources responsibly (e.g., to optimize therapeutic } \\
\text { benefit and sustain available supply) }\end{array}$ \\
\hline Trust & $\begin{array}{l}\text { Foster and maintain public, patient and healthcare provider } \\
\text { confidence in health system }\end{array}$ \\
\hline Utility & $\begin{array}{l}\text { Maximize the greatest possible good for the greatest possible } \\
\text { number of individuals with available resources }\end{array}$ \\
\hline
\end{tabular}

Figure 1. Guiding Ethical Principles for Managing Drug Shortages

Fifteen patients (both ICU and ward patients) in the surveyed week of March 9-15, 2021 did not receive an initial dose of tocilizumab because of clinician concerns around medication supply, and/or institutional policies that limited tocilizumab use (e.g., only to critically ill patients).

A further review indicated that a number of hospitals seeing patients with COVID-19 in Ontario were currently not on Ontario's allocation list for tocilizumab and they were unclear how these sites would access and administer tocilizumab to eligible patients. While only a few acute care teaching hospitals were not listed, $26 \%$ of large community hospitals and the majority of small community sites and rehabilitation sites were not allocated tocilizumab unless they were affiliated with a 
larger hospital network already on the list.

We have subsequently learned that at least 6 hospitals in Ontario have recently run out of tocilizumab supply and were unable to obtain additional supply through informal regional sharing.

\section{Can we optimize the distribution and supply of tocilizumab to ensure the most benefit?}

An ethical, evidence-based framework is required to inform allocation and use of limited tocilizumab supply. The Ontario COVID-19 Bioethics Table adapted a published Ontario drug supply shortage framework ${ }^{17}$ for use during the COVID-19 pandemic. ${ }^{18}$ It outlines a set of guiding ethical principles (Figure 1 ) and three stages for managing drug supply during the COVID-19 pandemic:

Stage 1: Preserve the standard of care for as many COVID-19 patients as possible by conserving supply, sharing supply, and procuring or accessing new supply.

Stage 2: Optimize therapeutic benefit based on existing evidence within available supply (Primary Allocation Principle).

Stage 3: Use a fair procedure to choose between COVID-19 patients (Secondary Allocation Principle) if Stage 1 and 2 efforts are insufficient to meet demand.

Guided by these ethical principles, informed by the rapid survey of providers described above, and acknowledging the increasing need of this scarce life-saving drug during Ontario's third wave of COVID-19, a set of recommendations has been developed to rationally allocate tocilizumab at each stage of the Ontario drug supply shortage framework.

\section{Recommendations and Options}

If Ontario enters a shortage of Tocilizumab, it will need to consider approaches that optimize the effectiveness and equity of the existing supply. Working from the Ontario COVID-19 Bioethics Table framework noted above, this Science Brief provides recommendations and options at each stage.

\section{All Stages of Managing Tocilizumab Supply and Distribution}

- Ontario should continue to preserve tocilizumab for CAR-T cell therapy and rheumatologic indications refractory to other therapy (supported by beneficence, equity, and stewardship). CPDN has mandated that existing supply for non-COVID patients be preserved, and not be utilized for COVID-19 therapy. However, efforts should be made to reduce the use of tocilizumab for rheumatological disorders if it can be done safely.

- Use of tocilizumab should follow the Ontario COVID-19 Science Advisory Table's Clinical Practice Guidelines, which are supported by randomized controlled clinical trials (supported by beneficence). The Ontario COVID-19 Science Advisory Table's Recommendation for use of tocilizumab accounted for the best available evidence in identifying COVID-19 patients with proven benefit from tocilizumab therapy: patients hospitalized with COVID-19 requiring at least supplemental oxygen who have not responded to initial dexamethasone therapy (representing a fraction of all patients infected with SARS-CoV-2). ${ }^{1}$ It is unclear if COVID-19 patients who are already mechanically ventilated derive greater benefit than those who have progressive disease and are not ventilated.

- Tocilizumab should be allocated province-wide to optimize therapeutic benefit and ensure equitable access for all eligible COVID-19 patients in Ontario 
(supported by beneficence, equity, reciprocity, solidarity, and trust).

\section{Options for All Stages of Managing Tocilizumab Supply and Distribution}

- The assessment of the stage of drug shortage should be based on predicted demand and communicated clearly to all clinical stakeholders (supported by beneficence, equity, and stewardship). Exponential growth of SARS-CoV-2 and changing public health measures in the community makes it difficult for most people to predict future tocilizumab needs. This requires mathematical modeling support to estimate the total future needs based on expected supply. If only ICU admissions are available, a reasonable estimate of all hospitalized COVID-19 patients (ICU and non-ICU) can be acquired by increasing this threefold (in the RECOVERY trial, the ratio of patients mechanically ventilated to those non-mechanically ventilated or without ventilatory support was approximately $1: 1)^{2}$ In Ontario, the ratio of patients in ICUs compared with those not in ICUs is approximately 1:1.2 to 1:1.6. Using this figure, a projection of demand in subsequent weeks can be made, with re-evaluation (and possibly re-calibration) weekly depending on observed usage.

- Ontario could create a near real-time provincial dashboard accessible to all clinical stakeholders that includes currently available tocilizumab supply, and allocated and administered doses (by region and site) which could also include any anticipated shipments of tocilizumab (supported by beneficence, equity, reciprocity, solidarity, and trust.) Having transparent data creates trust and a sense of solidarity, as all participants share in the need to preserve resources. Additionally, it makes it easier to recognize where some regions or sites might have substantially depleted tocilizumab supply, facilitating quicker responsiveness. This principle of a near-real time dashboard was why Ontario's Critical Care Information System (CCIS) was created: in 2003, Severe Acute Respiratory Syndrome (SARS) highlighted the need to improve Ontario's critical care system. In 2006, Ontario's Ministry of Health and Long-Term Care (now separated to $\mathrm{MOH}$ and MLTC) announced Ontario's Critical Care Strategy, and CCIS data collection began in 2007. CCIS data has been available throughout the COVID-19 pandemic to support monitoring and managing ICU bed and ventilator capacity. With tocilizumab, which may not be the last life-saving drug for which we will have insufficient supply, a similar approach needs to be rapidly established, but does not require patient-level data.

- Tocilizumab procurement could be provincial to support assessment of supply and management of supply (supported by equity). Allowing sites to contact CPDN directly or access tocilizumab through other distribution channels risks creating inequities among eligible patients in Ontario by allowing preferential access to tocilizumab.

- There could be a provincially coordinated mechanism building on the Incident Management Systems (IMS) to allow transfer of medication (or, rarely, patients) to ensure that any COVID-19 patient admitted to a hospital lacking tocilizumab supply can receive timely, evidence-based therapy (supported by equity and solidarity). Because of the sparse supply and cost of tocilizumab and the uneven distribution of COVID-19 patients across the province, patients may present to health care facilities that will not have access to tocilizumab.

\section{Recommendations for Stage $\mathbf{2}$ of Tocilizumab Supply and Distribution}

- The dose of tocilizumab should be reduced in line with the evidence in this brief. (supported by beneficence, equity, stewardship, and utility). As mentioned 
above, there are pharmacologic and clinical rationales for not administering a second dose, and for reducing the dose to a fixed dose of $400 \mathrm{mg}$, versus the usually higher $8 \mathrm{mg} / \mathrm{kg}$ weight-based dose (maximum $800 \mathrm{mg}$ ). This dose reduction would immediately increase drug supply for eligible Ontario COVID-19 patients.

- Triaging COVID-19 patients for tocilizumab therapy based on clinical factors deemed to be associated with benefit from therapy is not advisable for several reasons:

- The data are insufficient to define groups of COVID-19 patients most likely to benefit from treatment, especially because the treatment benefit appears to be greater with patients of milder initial illness. ${ }^{2}$ Denying COVID-19 patients with milder illness might increase the number of patients requiring mechanical ventilation.

- Although the relative risk reduction appears greater in younger individuals who have a lower overall risk of death, the absolute risk reduction is greater in older individuals. $^{2}$

\section{Options for Stage 2 of Tocilizumab Supply and Distributions}

- Distribution of tocilizumab could be weekly and site-specific, with allocation based on prior week's consumption (supported by equity, stewardship, and trust). With rapid changes in patient numbers, and potential redistribution of COVID-19 patients using an IMS, a prudent approach to distribution would be weekly distribution of tocilizumab to regions according to an IMS command structure. The IMS command structure can then distribute further based on local needs and supplies.

- Critical care triage guidelines are applicable to surges that occur in a short period of time, and allocation demands immediate choice. Additionally, ventilators cannot be saved today for use tomorrow. In Ontario, these triage guidelines (i.e., the Ontario Critical Care COVID-19 Command Centre's Emergency Standard of Care) cannot be applied unilaterally by a hospital and can only be applied at the direction of the IMS (i.e., Ontario's Critical Care COVID -19 Command Centre). A similar process is not applicable to the case of tocilizumab as planning for tocilizumab shortages allows allocation in a manner that has greater equity.

\section{Recommendations for Stage 3 of Managing Drug Shortages}

- Develop a logic-based mechanism to fairly ration tocilizumab (supported by beneficence, equity, solidarity, stewardship, trust, and utility). If Stage 1 and 2 efforts are insufficient to meet demand, Ontario will need to proceed to Stage 3, and use a fair procedure to choose between COVID-19 patients of equal need for secondary prioritization of tocilizumab. ${ }^{17,19}$ Stage 3 rationing should not occur on a site-by-site basis, but rather provincially, ideally managed through an IMS.

- Patients eligible for tocilizumab allocation are either moderately ill hospitalized patients (i.e., requiring supplemental oxygen via nasal prongs) or critically ill hospitalized patients (i.e., requiring oxygen via venturi mask, high-flow nasal cannula, non-invasive mechanical ventilation, invasive mechanical ventilation, or extra-corporeal membrane oxygenation (ECMO)) with suspected or confirmed COVID-19 who are on recommended doses of dexamethasone (or another doseequivalent corticosteroid) and are within 14 days of hospital admission or within 14 days of a new COVID-19 diagnosis if acquired in a healthcare setting. Moderately ill 
patients should have additional evidence of systemic inflammation, defined as a Creactive protein (CRP) of $75 \mathrm{mg} / \mathrm{L}$ or higher, and have evidence of disease progression (i.e., increasing oxygen or ventilatory requirements) while on dexamethasone or another dose-equivalent corticosteroid for treatment of COVID19. ${ }^{1}$ Moderately ill COVID-19 patients at hospitals not offering CRP measurement should be considered for tocilizumab allocation.

- Tocilizumab should be given to COVID-19 patients with a reasonable chance of benefiting from it. Because of the absolute scarcity, it is defensible not to offer tocilizumab to COVID-19 patients who almost certainly will not benefit. This should be based on consensus among those involved in assessing eligibility for tocilizumab allocation.

- A strictly first-come, first-served approach is not recommended, as it would favour patients presenting to centres with known tocilizumab supply, could potentially encourage early treatment of patients with milder disease, and would also benefit those in urban centres (with existing or ready supply) over rural locations.

- Drug administration should continue to be guided by supporting evidence, and COVID-19 patients who meet the eligibility criteria but do not want active medical treatment or specific treatment with tocilizumab should neither be offered it nor entered into an allocation system.

\section{Options for Stage 3 of Tocilizumab Supply and Distribution}

- To mitigate potential bias influencing the assessment of COVID-19 patients eligible for tocilizumab, a second-opinion or consensus model among those responsible for assessing eligibility should be considered.

- An allocation lottery could be the fair default procedure for choosing among eligible COVID-19 patients where allocation based on maximizing benefits cannot be determined. Allocation lotteries have been successfully used in several North American jurisdictions and acknowledges that the efforts to allocate based on maximizing the benefits of therapy have been exhausted. An added potential benefit of an allocation lottery framework is that it could track outcomes of COVID-19 patients offered or declined access to tocilizumab therapy. This would allow for evaluation of real-world use in Ontario, given our current recommendation for tocilizumab's place in standard of care and rising prevalence of variants of concern (VOC). ${ }^{20}$

- Because supply of tocilizumab should be seen as a provincial resource, if implemented, the allocation lottery should be initiated and managed centrally to avoid inequities in access due to geography. Otherwise, there is a risk that COVID-19 patients presenting to smaller or remote sites would be unfairly disadvantaged by the lottery system.

- A committee should oversee the allocation lottery procedure, with representation of medical specialties prescribing tocilizumab, regional considerations, and patient and caregiver representation from communities affected by allocation.

- An ideal allocation lottery would ensure meaningful and individualized access to tocilizumab for all COVID-19 patients, would avoid discrimination, and would mitigate disparities in outcomes due to social inequalities. Some jurisdictions are using a weighted lottery or categorical reserve system, as described and established by the University of Pittsburgh, ${ }^{21}$ to address both equity and reciprocity considerations; individuals from disadvantaged areas and essential 
workers are given heightened priority points in the lottery. COVID-19 patients who are essential workers should be given heightened priority, not because they are intrinsically more worthy, but because of their instrumental value to save others and ensure the continuity of critical societal infrastructure. This heightened priority may also be justified by a reciprocal obligation to provide treatment to individuals who are at heightened risk in occupations needed to safeguard society during the pandemic. It is important to note that the category of essential workers includes not only health care workers but also workers who may be socially and economically vulnerable, such as grocery workers, transportation workers, agricultural workers, childcare workers and custodial workers. Therefore, giving essential workers heightened access to treatment may also mitigate the disproportionate impact of COVID-19 on disadvantaged communities. Such a model requires time-consuming broad stakeholder engagement to discuss the value laden dimensions of a weighted lottery. At this juncture, such a model is likely not feasible in Ontario. A simple unweighted lottery is therefore recommended.

- The first step to an allocation lottery would be to predict the anticipated number of COVID-19 hospitalized cases and the available doses of tocilizumab over a defined time period, and to decide what percentage of eligible patients should receive tocilizumab. (Figure 2). If, for example, there is enough tocilizumab over a 4 -week period for $40 \%$ of eligible patients, patients would then be entered in a lottery with a $40 \%$ chance of selection. This scheme should be revisited regularly (e.g., weekly) to ensure optimal use of tocilizumab.

Lottery Allocation for Treatment of COVID-19 Patients with Tocilizumab

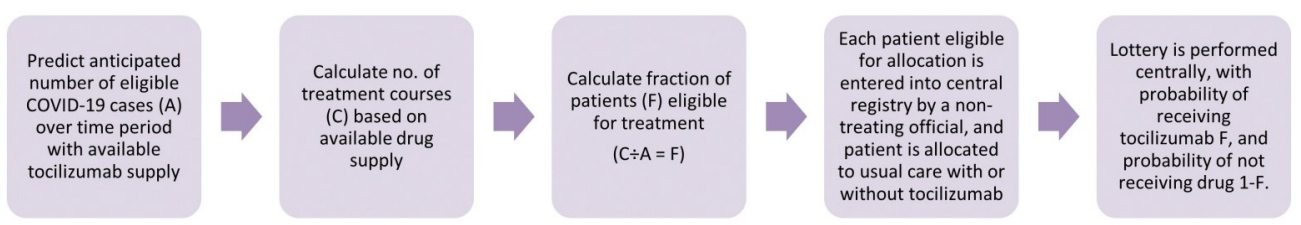

Figure 2. Process to Calculate and Allocate COVID-19 Patients to Tocilizumab Using a Non-Weighted Lottery Prior to implementation, the process requires a method of estimating the number of hospitalized COVID-19 patients who are eligible for tocilizumab therapy, and a Provincial Dashboard with real-time information regarding tocilizumab supply, including hospital site, quantity, and vial sizes. A central registry for allocation is necessary. Ideally, data are entered into the central registry by a non-treating hospital official when COVID-19 patients are being considered for treatment. The registry would then provide immediate results of the lottery, i.e., whether the patient was allocated to receive or not receive tocilizumab in addition to usual care.

- Information collected at lottery registration should include: patient demographic information, prescriber information, and site of care. Consideration should be given to minimize information required from the person entering the information. It should also account for patients not having an eligible health card number (to maximize equity), and should avoid requiring information that might create bias. This information should be entered by a designated hospital official, rather than the treating physician.

- Use of other COVID-19-specific therapy (i.e., systemic corticosteroids and remdesivir) should be entered as well, including start dates.

- After patients are registered, the hospital official would receive treatment allocation following randomization, and would report the results of randomization to the prescriber and to the hospital pharmacy supplying tocilizumab. If the hospital does not have tocilizumab, they would notify IMS to arrange delivery of a dose of tocilizumab. 
- Implementing such a strategy would require careful communication. The communication strategy should include separate messaging to hospital administrators and health care leaders, health care providers, patients and their families, and the public (including scripts to support health care providers as they communicate with patients and their families).

- Patient-centred outcomes would be evaluated to assess the effectiveness of allocation and treatment during the shortage and under the new guidelines.

- Ideally, the allocation system could also be used to test therapeutic hypotheses (i.e., a pragmatic clinical trial comparing $400 \mathrm{mg} v \mathrm{vs} .800 \mathrm{mg}$ tocilizumab dosing). ${ }^{22}$

\section{Interpretation}

Tocilizumab demand in Ontario might exceed supply in the near future. A scienceand ethics-guided approach designed to maximize the benefits of available supply is recommended.

Core recommendations include optimizing the dose of tocilizumab to a single intravenous dose of $400 \mathrm{mg}$ for all patients; considering use of sarilumab as a substitute for tocilizumab; establishing a provincial dashboard of tocilizumab supply and distribution accessible to all stakeholders; and ongoing modeling to estimate the supply-to-demand adequacy.

As a default, a centralized allocation lottery system should be employed as soon as predicted demand exceeds supply. Other aspects may need to be taken into account for allocation, as appropriate.

\section{Methods Used for This Science Brief}

We searched PubMed and Google Scholar using the following search terms: "IL-6 inhibitor", "interleukin-6 inhibitor", "tocilizumab" and "sarilumab". In addition, we retrieved reports citing relevant articles through Google Scholar and reviewed references from identified articles for additional studies. The search was last updated on April 5, 2021. Expert perspectives were sought from critical care medicine, medical ethics, internal medicine, and pharmacy in Ontario, and we also contacted experts from the United States (JAJ and EKM) who had designed and/or implemented state-wide drug randomization during the COVID-19 pandemic, to provide us with detailed information on various issues around implementation of weighted and unweighted drug lottery systems.

For tocilizumab therapeutic recommendations, we used the following definitions for COVID-19 disease severity:

\section{Critically III}

Patients requiring ventilatory and/or circulatory support, including high-flow nasal oxygen, non-invasive ventilation, invasive mechanical ventilation, or extracorporeal membrane oxygenation (ECMO). These patients are usually managed in an intensive care setting.

\section{Moderately III}

Patients newly requiring low-flow supplemental oxygen. These patients are usually managed in hospital wards.

\section{Mildly III}

Patients who do not require new or additional supplemental oxygen from their 
baseline status, intravenous fluids, or other physiological support. These patients are usually managed in an ambulatory/outpatient setting.

\section{Author Contributions}

AMM conceived the Science Brief and wrote the first draft. All authors revised the Science Brief critically for important intellectual content and approved the final version.

The authors would like to thank members of Ontario's Science Advisory Table Drugs \& Biologics Clinical Practice Guidelines Working Group for their contribution to optimizing the supply of tocilizumab by using an altered dosing regimen in this Science Brief. The authors would also like to thank the members of the University of Toronto Joint Centre for Bioethics for their contributions to establishing ethical principles in the event of drug shortages.

\section{References}

1. Morris AM, Stall NM, Bobos $P$, et al. Tocilizumab for Hospitalized Patients with COVID-19.; 2021. https://doi.org/10.47326/ocsat.2021.02.11.1.0

2. Horby PW, Pessoa-Amorim G, Peto L, et al. Tocilizumab in patients admitted to hospital with 3 COVID-19 (RECOVERY): preliminary results of a 4 randomised, controlled, open-label, platform trial. medRxiv. Published online 2021. https:// doi.org/10.1101/2021.02.11.21249258

3. Investigators, Remap-Cap, Gordon AC, Mouncey PR, et al. Interleukin-6 Receptor Antagonists in Critically III Patients with Covid-19. N Engl J Med. Published online 2021. https://doi.org/10.1056/NEJMoa2100433

4. Frey N, Grange S, Woodworth T. Population pharmacokinetic analysis of tocilizumab in patients with rheumatoid arthritis. J Clin Pharmacol. 2010;50 (7):754-766.

5. Dhillon S. Intravenous tocilizumab: a review of its use in adults with rheumatoid arthritis. BioDrugs. 2014;28(1):75-106.

6. Shetty A, Hanson R, Korsten $\mathrm{P}$, et al. Tocilizumab in the treatment of rheumatoid arthritis and beyond. Drug Des Devel Ther. 2014;8:349-364.

7. Yang C, Liu M. Tocilizumab in Treatment for Patients With COVID-19. JAMA Intern Med. Published online April 5, 2021. https://doi.org/10.1001/ jamainternmed.2021.0392

8. Bastida C, Ruiz-Esquide V, Pascal M, et al. Fixed dosing of intravenous tocilizumab in rheumatoid arthritis. Results from a population pharmacokinetic analysis. Br J Clin Pharmacol. 2018;84(4):716-725.

9. Del Valle DM, Kim-Schulze $\mathrm{S}$, Huang $\mathrm{H}-\mathrm{H}$, et al. An inflammatory cytokine signature predicts COVID-19 severity and survival. Nat Med. 2020;26(10):16361643.

10. Strohbehn GW, Heiss BL, Rouhani SJ, et al. COVIDOSE: A Phase II Clinical Trial of Low-Dose Tocilizumab in the Treatment of Noncritical COVID-19 Pneumonia. Clin Pharmacol Ther. 2021;109(3):688-696.

11. Comparison of Tocilizumab Versus Tocilizumab/Infliximab in Patients With COVID-19-associated Cytokine Storm Syndrome. Accessed April 8, 2021. https:// clinicaltrials.gov/ct2/show/NCT04734678? term=tocilizumab+coronavirus \& recrs $=a \& d r a w=2 \&$ rank $=3$

12. Favipiravir Combined With Tocilizumab in the Treatment of Corona Virus 
Disease 2019. Accessed April 8, 2021. https://clinicaltrials.gov/ct2/show/ NCT04310228?term=tocilizumab+coronavirus\&recrs=a\&draw=2\&rank=1

13. Murthy S, Lee TC. IL-6 blockade for COVID-19: a global scientific call to arms. Lancet Respir Med. Published online March 4, 2021. https://doi.org/10.1016/ S2213-2600(21)00127-2

14. REMAP-CAP Investigators. Equivalency of tocilizumab and sarilumab. REMAPCAP. Published April 10, 2021. Accessed April 11, 2021. https:// www.remapcap.org/covid19publications

15. Product Monograph Including Patient Medication Information: Kevzara (sarilumab). Sanofi-aventis Canada Inc.; 2017. https://healthproducts.canada.ca/dpd-bdpp/ info.do;jsessionid=7C4436289151CEEB75EF2912B729D342? lang=en\&code $=96232$

16. Science Advisory and Modelling Consensus Tables. Update on COVID-19 Projections (April 1, 2021). Ontario COVID-19 Scientific Advisory Table; 2021. https://covid19-sciencetable.ca/sciencebrief/update-on-covid-19-projections-5/

17. Gibson JL, Bean S, Chidwick P, Godkin D, Sibbald RW, Wagner F. Ethical framework for resource allocation during a drug supply shortage. Healthc Q. 2012;15(3). https://doi.org/10.12927/hcq.2013.23040

18. Bean S, Denburg A, Greenberg R, Anderson J. Ethical Framework for Drug Shortages That Occur during the COVID-19 Pandemic in Ontario. Joint Centre for Bioethics, University of Toronto; 2020. https://jcb.utoronto.ca/wp-content/ uploads/2021/04/Ethical-Framework-for-Drug-Shortages-during-COVIDPandemic.pdf

19. Emanuel EJ, Persad G, Upshur R, et al. Fair Allocation of Scarce Medical Resources in the Time of Covid-19. N Engl J Med. 2020;382(21):2049-2055.

20. Tuite AR, Fisman DN, Odutayo A, et al. COVID-19 Hospitalizations, ICU Admissions and Deaths Associated with the New Variants of Concern. Ontario COVID-19 Science Advisory Table; 2021. https://doi.org/10.47326/ ocsat.2021.02.18.1.0

21. A model hospital policy for fair allocation of medications to treat COVID-19. Accessed April 3, 2021. https://ccm.pitt.edu/node/1133

22. Reid R. Embedding research in the learning health system. Healthcare Papers. 2016;16:30-35. 